\title{
Sistema nacional de informações tóxico-farmacológicas: o desafio da padronização dos dados
}

\author{
National poison information system: \\ the challenge of the data standardization
}

Rosane Abdala Lins de Santana ${ }^{1}$

Rosany Bochner ${ }^{1}$

M aria Cristina Soares Guimarães ${ }^{1}$

${ }^{1}$ Laboratório de Pesquisa em Ciência, Tecnologia e Inovação em Saúde,

Instituto de Comunicação e Informação Científicae

Tecnológica em Saúde

Fundação O swaldo Cruz.

Av. Brasil 4.365/Pavilhão

H aity M oussatché/204,

M anguinhos. 21040-900

Rio deJaneiro RJ.

rabdala@icict.fiocruz.br
Abstract Human poisoning is a serious public health issue around the world. Readily available and reliableinformation in this field are essential to the full implementation of epidemiological and sanitary surveillanceand to establish efficient and effective strategies of control. The continuous improvement of data quality and consistency \% no doubt, a permanent challenge $\%$ has a key role in the definition of public policies. To assess the accuracy of Brazilian data on human poisonings, this study explores data from a network of Toxicological Information and AssistanceC enters, one of the main sources of such data in Brazil. Data are consolidated by the $\mathrm{N}$ ational Poison Information System (SI NITOX). Assuming that standardization is one of the main procedures that influence the data quality collected and analyzed by SIN ITOX, the study assesses the operations of the Centers affiliated to the SI N ITOX in charge to interpret and register the events reported to them and highlights the procedures used to standardize case reports. The results show an evident insufficient degree of standardization of the data received at SINITOX. We emphasize the need to design strategies to improve standardization, aiming to develop a reliable system and to minimize the harms associated to human poisoning in Brazil. Key words Standardization, Data quality, Human poisoning, Information system
Resumo A incidência de intoxicações e envene namentos constitui um grave problema de saúde pública. Para esse segmento, a importância de se dispor, a tempo e a hora, de informações fidedignas é condição primordial para se traçarem estratégias eficazes e efetivas de vigilância epidemiológica esanitária. Para investigar uma faceta da qualidade dos dados registrados no país, sobreeste tipo de agravo, este estudo volta-se para os Centros de Informação e Assistência Toxicológica (CIAT), uma das principais fontes para notificação e registro de tais casos, que fornecem dados que são consolidados pelo Sistema Nacional de Informações Tóxico-Farmacológicas (SI NITOX). Partindo do pressu posto quea padronização éuma das principais variáveis que impactam a qualidade dos dados coletados, o estudo vi sou analisar a maneira como os CIAT interpretam e registram cada um dos eventos a eles notificados. Buscou-se reproduzir as práticas em curso no registro dos casos, com sua lógica e entendimento habitual. Os resultados apontam para o baixo grau de padronização dos dados que chegam ao SI N ITOX, 0 que impacta, qualitativamente, as informações geradas por este sistema. U m desenho de estraté gias que atuem para aprimorar a padronização dos dados, por certo, conduzirá a um sistema de informação mais robusto e confiável.

Palavras-chave Padronização, Qualidade de dados, Intoxicação, Sistema de informação 


\section{Introdução}

$\mathrm{Na}$ área da saúde, a incidência de intoxicações e envenenamentos, no Brasil, como no resto do mundo, constitui um grave problema de saúde pública; para mudar esse quadro, as autoridades responsáveis por este setor precisam de informações fidedignas para que possam nortear suas ações.

Especialmente a partir da década de noventa do século passado, pesquisadores afirmam que os casos de envenenamentos decorrentes da falta de orientação da população sobre os produtos químicos disponíveis no mercado e seu uso terapêutico vêm se multiplicando no Brasil ${ }^{1}$. Os autores chamam atenção para o risco causado pela desinformação a respeito das drogas (inclusive as medicinais) que estão acessíveis aos consumidores, além dos aditivos alimentares até os inseticidas agrícolas, passando por medicamentos, cosméticos e remédios da chamada flora medicinal.

A importância de se dispor de informações confiáveis sobre a incidência de intoxicações no país, no tempo hábil para tomada de decisão, é condição primordial para que os tomadores de decisão possam delinear estratégias de ação para as vigilâncias epidemiológica e sanitária. Segundo M ota et al. ${ }^{2}$, As atividades de vigilância epidemiológica no monitoramento permanente da situação de saúde necessitam de informações que permitam surpreender o mais precocemente possível as mudanças nos padrões de morbidade e mortalidade e indicar as medidas de controle pertinentes. A permanente atualização das informações, a sua qualidade ea disponibilidade oportuna são fatores importantes para decisão e a ação correspondente.

$N$ a perspectiva de Angeloni ${ }^{3}$, informações são geradas a partir de dados, que constituem sua matéria-prima. Dados são conceituados como elementos brutos, sem significado e desvinculados da realidade. Por decorrência, ena perspectiva do presente trabalho, se informação é produzida pelo processo de contextualização e análise de dados, a qualidade da informação gerada é função da qualidade do dado primário, ou, do registro efetuado em sua fonte original.

No caso das informações sobre intoxicações e envenenamentos, parte significativa dos dados primários é gerada nos Centros de Informação e Assistência Toxicológica (CIAT), unidades organizacionais de diferentes perfis e estruturas, dispersas geograficamente pelo país. Segundo a Agência N acional deVigilância Sanitária (AN VI$\mathrm{SA})^{4}$, os $\mathrm{CIAT}$ são unidades especializadas que têm a função de fornecer informação e orienta- ção sobre diagnóstico, prognóstico, tratamento e prevenção das intoxicações, assim como a toxicidade das substâncias químicas e biológicas, e os riscos que elas oferecem à saúde, bem como prestar assistência ao paciente intoxicado.

Além dessas atribuições, cabe aos CIAT o registro dos dados de intoxicação eenvenenamento. 0 registro pode ser feito tanto pelo atendimento pessoal do paciente, ou simplesmente por meio da prestação de uma informação, seja de forma pre sencial ou via telefone. Ambas as situações pedem o registro ou notificação, em uma ficha padronizada pela rede de centros. É também facultado o registro em outros formatos de formulários, adaptados ou customizados, tendo em vista as necessidades e peculiaridades de cada CIAT.

Os dados coletados são posteriormente enviados para o Sistema Nacional de Informações Tóxico-Farmacológicas (SINITOX), onde são consolidados.

Portanto, o estudo visa analisar a maneira como os CIAT interpretam e registram cada um dos eventos a eles notificados, dentro de uma abordagem metodológica que buscou ressaltar a importância da padronização nos processos de registro dos casos.

\section{M etodologia}

Para ganhar entendimento sobre a precisão do instrumento de coleta de dados usado pel os CIAT no registro dos casos de intoxicação, foi desenvolvido um estudo de caso. Segundo Yin ${ }^{5}$, um estudo de caso é "uma inquirição empírica que investiga um fenômeno contemporâneo dentro de um contexto da vida real", no qual os comportamentos relevantes não podem ser manipulados, ecaracteriza-sepela"capacidade delidar com uma completa variedade de evidências - documentos, artefatos, entrevistas e observações". Para Stake ${ }^{6}$, a ênfase dessa perspectiva metodológica está na compreensão, fundamentada basicamente no conhecimento tácito, o que tem uma forte ligação com intencionalidade, o que não ocorre quando o objetivo é meramente explanação, baseada no conhecimento proposicional. Erskine et al. ${ }^{7}$ definem um caso como a descrição de uma situação recente, que envolve uma decisão ou um problema. Ele normalmente é proposto sob o ponto de vista daquele que está envolvido com a decisão e permite 0 acompanhamento e análise dos passos de quem tomou a decisão.

No presente trabalho, o estudo de caso envolveu a construção de um "protocolo" para co- 
leta dos dados, em que se buscou contextualizar uma "situação problema" como uma situação vivida pel os sujeitos da pesquisa, o que justifica a inclusão de dados subjetivos relacionados à visão que as pessoas envolvidas na situação têm da mesma. Assim, com a orientação de um pesquisador sênior do Centro de Informação Toxicológica do Rio Grande do Sul, e baseadas em fatos passados reais, foram montadas e narradas situações hipotéticas de intoxicação/envene namento, em um total de vinte casos. Os mesmos foram enviados aos CIAT com a solicitação de que fossem registrados em seus formulários de notificação de acordo com sua lógica e entendimento habitual. Guardadas as limitações intrínsecas a essa abordagem metodológica, o que se buscou real çar é a importância da padronização dos dados para maximizar a representatividade dos eventos no sistema, com um alerta por sobre as práticas, subjetividades e contingências locais, que são elementos que somam complexidade a fenômenos por si só já multifacetados.

Quando da realização do estudo aqui descrito, o Brasil contava com 35 CIAT na formação da chamada Rede $\mathrm{N}$ acional de Centros de Informação e Assistência Toxicológica, que cobria de zoito Estados e o Distrito Federal, com uma distribuição irregular ao longo do país. Desse universo, trinta centros concordaram em participar da pesquisa. Foi solicitado a cada centro participante que procurasse registrar os casos, seguindo os procedimentos que são uti lizados segundo sua rotina de trabalho, procurando assegurar e reproduzir, de forma mais fidedigna possível, a realidade da prática cotidiana.

A partir da ficha de notificação e de atendimento (doravante chamada ficha), instrumento padrão para coleta de dados nos centros, foram el eitas as seguintes variáveis para controle: agentetóxico envolvido, circunstância deocorrênciae via de exposição/intoxicação. Essas variáveis foram escolhidas por apresentarem, em sua natureza semântica, um maior grau de subjetividade, com potencial para ocasionar interpretações dúbias no ato de preenchimento da ficha.

A análise dos resultados foi realizada tomando como guia as Monografias de Toxicologia de Urgência (M TU), publicação "que se propõe de forma objetiva e prática a atualizar ea padronizar as informações técnicas, disponíveis na área da Toxicologia Clínica de Urgência, para a Rede de Centros de Assistência Toxicológica". O utra fonte utilizada foi o $\mathrm{M}$ anual dePreenchimento da Ficha de $\mathrm{N}$ otificação e de Atendimento ${ }^{9}$, que visa esclarecer o preenchimento da ficha de notificação.
Ambos os documentos são fontes de consulta que deveriam ser utilizadas para apoiar as decisões tomadas pelos profissionais dos centros no preenchimento dos registros quando do atendimento dos casos de intoxicação/envenenamento.

Com o objetivo de melhor conhecer a realidade dos centros, além do envio dos casos para registro, foram realizadas entrevistas em quatro centros, escolhidos por se localizarem em três diferentes regiões do país e por possuírem características e realidades diferentes: Serviço de Toxicologia de M inas Gerais, Centro de Informação Toxicológica do Rio Grande do Sul, Centro de Informação Toxicológica de Belém e Centro de Controle de Intoxicação de $\mathrm{N}$ iterói.

A entrevista foi composta por três blocos de questões, dirigidas a profissionais que desempenhavam diferentes funções nos centros. A primeira ea segunda parte, dados do centro e coordenação do centro, foram direcionadas ao coordenador do centro, e tinham como objetivo entender o contexto de sua criação e conhecer sua infra-estrutura; a terceira parte, preenchimento da ficha de notificação, dirigida ao profissional que habitual mente preenchea ficha, quando procede ao atendimento de um caso de intoxicação/ envenenamento, seja presencial, seja via tel efone. Procurou-se, aqui, explicitar a prática do processo de notificação desse caso.

São apresentadas a seguir, a título de esclarecimento, al gumas definições usadas no presente estudo, oriundas do Manual de Preenchimento da Ficha de N otificação e de Atendimento" ${ }^{9}$ :

- Variável: considera-se como variável o agente tóxico envolvido no caso, a circunstância de ocorrência e a via da exposição/intoxicação que foram questionadas no estudo de caso;

- Categoria: considera-se como categoria as classificações adotadas em cada variável, deacordo com a ficha de notificação e atendimento que os centros utilizam. São elas: (a) categorias da variável agente tóxico: medicamentos, agrotóxicos de uso agrícola, agrotóxicos de uso doméstico, produtos veterinários, raticidas, domissanitários, cosméticos, produtos químicos industriais, metais, drogas de abuso, plantas, alimentos, animais peçonhentos/serpentes, animais peçonhentos/aranhas, animais peçonhentos/ escorpiões, outros animais peçonhentos/venenosos, animais não peçonhentos, desconhecido e outros; (b) categorias da variável circunstância: acidente individual, acidente col etivo, acidenteambiental, ocupacional, uso terapêutico, prescrição médica inadequada, erro de administração, automedicação, abstinência, abuso, ingestão deali- 
mentos, tentativa de suicídio, tentativa de aborto, violência/homicídio, uso indevido, ignorada e outra; (c) categorias da variável via: oral, cutânea, respiratória, parenteral, nasal, ocular, retal, vaginal, mordedura/picada, ignorada e outra.

Para fins deste artigo, são reportados, a seguir, aqueles casos que geraram maior discordância de categorização por parte dos centros.

\section{Resultados}

A pesquisa contou com um índice de retorno de $80 \%$, ou seja, dos trinta questionários enviados, participaram da análise 24 centros, os quais foram responsáveis por aproximadamente $89,0 \%$ e $94,4 \%$ das notificações dos casos de intoxicação/ envenenamento humanos publicadas pelo SI NITOX nos anos de 2001 e 2002, respectivamente.

Os centros que participaram da pesquisa analisaram cada caso que compõem o questionário e assinalaram a categoria pertinente para cada uma das variáveis consideradas, agente tóxico, circunstância evia deocorrência, de acordo com seu entendimento e interpretação.

0 ideal seria quetodos os centros registrassem cada um dos casos da mesma forma, ou seja, assinalassem as mesmas categorias para as variáveis consideradas. Porém, isso não ocorreu, como se verifica nas descrições a seguir, nas quais são detaIhados, para cada uma das variáveis, os casos que apresentaram maior divergência entre os centros, ou seja, casos em que um número maior de categorias foi assinalado para cada uma das variáveis.

\section{Variável agente tóxico}

Em relação a esta variável, somente em três dos vinte casos os centros tiveram o mesmo entendimento da situação apresentada ao assinalar a mesma categoria. Por outro lado, em três outros casos, a não concordância dos centros pode ser observada pelo aparecimento de cinco categorias diferentes para um mesmo caso hipotético.

O Quadro 1 apresenta as categorias da variável agente tóxico assinaladas pelos centros e respectivas frequências, para os três casos que apresentaram maior discordância entre os centros.

No caso 1, pode-se observar que a intoxicação foi originada pela mistura de $N$ eosal dina com álcool. De acordo com as Monografias em Toxicologia de Urgência (MTU) $)^{8}$, a N eosaldina é um medicamento eo álcool etílico (etanol) éclassificado como um produto químico industrial, independente de seu uso, o que parece ser questionável pelos centros, uma vez que nenhum deles assinalou a categoria medicamento + produto químico industrial.

Quadro 1. Descrição dos três casos que apresentaram maior discordância entre os centros para a variável agente tóxico.

\begin{tabular}{|l|l|c|}
\hline \multicolumn{1}{|c|}{ Relato dos casos } & \multicolumn{1}{|c|}{ Categorias do agente tóxico } & Número de Centros \\
\hline Caso 1. J. M ., 17 anos de idade, feminino, 55 & M edicamentos & 9 \\
kg. Paciente ingeriu na tentativa de suicídio 10 & Medicamentos + drogas de abuso & 9 \\
comprimidos de N eosal dina mais quantidade & Medicamentos + domissanitários & 4 \\
ignorada de álcool (etanol). Chega ao pronto- & Produtos químicos industriais & 1 \\
socorro embriagada. Sinais vitais estáveis. & Drogas de abuso & 1 \\
\hline Caso 2. T. G., 5 anos de idade, feminino, 13 & Medicamentos & 16 \\
kg. M ãe aplicou na cabeça da criança produto & Agrotóxicos/uso doméstico & 5 \\
à base de deltametrina e deixou por 30 min. & Produtos veterinários \\
(indicado 10 min.). A criança começou a & Domissanitários & 1 \\
apresentar vômitos. & Metais & 1 \\
& & 1 \\
\hline Caso 3. R. M., 4 anos de idade, masculino. & Metais & 11 \\
M enino ingeriu pilha de bateria para relógio. & Produtos químicos industriais & 7 \\
& Outro* & 3 \\
& Domissanitários & 2 \\
& Produtos químicos industriais + & 1 \\
\hline
\end{tabular}

*Não especificada. 
Com relação ao caso 2, a deltametrina, segundo as $\mathrm{MTU}^{8}$, éum inseticida piretróide, classificado como agrotóxico de uso agrícola. Contudo, consiste também no princípio ativo de alguns medicamentos utilizados para o tratamento da pediculose, o que pode explicar o grande número de centros que assinalaram a categoria "medicamentos". Por outro lado, oito centros não tiveram o mesmo entendimento e, dentre estes, cinco consideraram a categoria "agrotóxicos de uso doméstico".

Com relação ao caso 3, a classificação encontrada para pilha de bateria nas M TU ${ }^{8}$ foi "miscelânea", ou seja, uma mistura. É composta por uma parte de solução eletrolítica e uma parte de quantidadevariável de metal pesado. A categoria mais assinalada foi "metais" seguida por "produtos químico industriais".

\section{Variável circunstância}

Em relação a esta variável, somente em dois dos vinte casos os centros apresentaram concor- dância perfeita, ou seja, assinalaram a mesma categoria. Por outro lado, em três outros casos a não concordância dos centros pode ser observada pelo aparecimento deoito, sete eseis categorias diferentes para cada um dos casos hipotéticos apresentados.

O Quadro 2 apresenta as categorias da variável circunstância assinaladas pel os centros erespectivas frequências, para os três casos que apresentaram maior discordância entre os centros.

0 caso 4 gerou muita polêmica na classificação da circunstância. De acordo com o M anual de Preenchimento da Ficha de N otificação e de Atendimento ${ }^{9}$, as duas categorias entre as três mais assinaladas pelos centros, "uso terapêutico" e "automedicação" são exclusivas de medicamentos, e este caso envolve uma planta.

Das outras categorias que tiveram um menor número de marcações, chama a atenção "acidente coletivo", que segundo o mesmo manual é definido como "qualquer caso de intoxicação e/ ou exposição não intencional por qualquer produto e/ou substância química em mais de uma

Quadro 2. Descrição dos três casos que apresentaram maior discordância entre os centros para a variável circunstância.

\begin{tabular}{|l|l|c|}
\hline \multicolumn{1}{|c|}{ Relato dos casos } & \multicolumn{1}{|c|}{ Categorias da circunstância } & Número de Centros \\
\hline Caso 4. W.T.., 52 anos de idade, feminino. & Uso terapêutico & 6 \\
Paciente fez chá à base de arruda. Passou a & Auto medicação & 5 \\
apresentar diarreia e vômitos & Uso indevido & 5 \\
& Acidente individual & 2 \\
& Ignorada & 2 \\
& Acidente coletivo & 1 \\
& Ingestão de alimentos & 1 \\
& Outra * & 1 \\
\hline Caso 5. E. P., 35 anos de idade, masculino. & Não considerado intoxicação & 1 \\
Acometido de doença reumática em seu & Uso indevido & 9 \\
cotovelo esquerdo, vai a curandeiro e se deixa & Outra *idente individual & 4 \\
ser picado por 50 abelhas no mesmo cotovelo. & Uso terapêutico & 3 \\
Após, apresentou choque, sendo levado ao & Auto medicação & 3 \\
hospital do pronto-socorro, onde foi medicado & Violência/homicídio & 3 \\
eliberado. & Uso terapêutico + uso indevido & 1 \\
\hline Caso 6. M.J.G., 38 anos de idade, feminino. & Auto medicação & 1 \\
Passou creme esfoliante na pele do rosto como & Uso terapêutico & \\
tratamento antirrugas indicado por amiga. & Outra *** & \\
Apresenta edema, eritema e ardência na área & Acidente individual & 7 \\
em que utilizou o produto. & Uso indevido & 5 \\
& Uso terapêutico + automedicação & \\
\hline
\end{tabular}

${ }^{*}$ Não especificada; ** M eios alternativos por crendice popular, prática de curandeirismo, 2 não especificadas; ${ }^{* * *}$ Indicação leiga, efeito adverso, reação adversa a medicamento, uso adequado, não especificada. 
vítima". Em nenhum momento o caso anteriormente ilustrado mostra a participação de mais de uma pessoa; portanto, deduz-se que esta categoria foi marcada por engano.

No caso 5, a categoria "uso indevido" foi a mais assinalada, seguida de "outra". As categorias "uso terapêutico" e "automedicação", específicas de medicamentos, já mencionadas anteriormente, mais uma vez são usadas fora do contexto.

No caso 6, as duas categorias entre as três mais assinaladas pelos centros, "automedicação" e"uso terapêutico" são exclusivas demedicamentos e este caso envolve um cosmético.

$\mathrm{Na}$ categoria "outra", uma especificação foi "uso adequado", que mostra mais uma vez o pouco cuidado na notificação do caso, pois se o uso foi adequado, por que ocorreu a intoxicação?

\section{Variável via de ocorrência}

Esta variável apresentou o maior número de casos em que os centros tiveram o mesmo entendimento, ou seja, em dez dos vinte casos os centros assinalaram a mesma categoria para a via de ocorrência.

O Q uadro 3 apresenta as categorias da variável via de ocorrência assinaladas pelos centros e respectivas frequências, para os três casos que apresentaram maior discordância.
No caso 7, apesar de apresentar quatro categorias, a via cutânea foi assinalada por vinte dos 24 centros. Contudo, chama a atenção o fato da via oral ter sido assinalada por dois centros, pois em nenhum momento foi citado que a criança ingeriu o produto.

Em "outra", foi especificado "via couro cabeludo" e, neste caso, o manual não define cada uma das vias de exposição e/ou intoxicação que a ficha de notificação possui. Dessa forma, deixa vago se couro cabeludo pode ou não ser considerado "via cutânea".

No caso 8, a classificação "outra" recebeu o maior número demarcações, o quechama a atenção. $\mathrm{Na}$ especificação dos dezenove centros em relação a essa classificação, eles foram praticamente unânimes em apresentar como sugestão algo relacionado à via placentária.

A pesar de apresentar quatro categorias diferentes, no caso 9, vinte dos centros participantes do estudo tiveram o mesmo entendimento, assinalando a via oral.

\section{Discussão}

A padronização é um processo muito importante, quando se trata de atividades que envolvem vários centros, com perfis erealidades diferentes.

Quadro 3. Descrição dos três casos que apresentaram maior discordância entre os centros para a variável via de ocorrência.

\begin{tabular}{|l|l|r|}
\hline \multicolumn{1}{|c|}{ Relato dos casos } & \multicolumn{1}{|c|}{ Categorias da via } & Número de Centros \\
\hline $\begin{array}{l}\text { Caso 7. T. G., } 5 \text { anos de idade, feminino, 13 kg. M ãe } \\
\text { aplicou na cabeça da criança produto à base de } \\
\text { deltametrina e deixou por 30 min. (indicado 10 }\end{array}$ & Cutânea & 20 \\
min.). A criança começou a apresentar vômitos. & Respiratória & 2 \\
\hline Outra * & 1 \\
\hline Caso 8. Recém-nascido, 2,8 kg. A mãe do recém- & Outra ** & 1 \\
nascido é usuária crônica de cocaína. A criança & Parenteral & 19 \\
apresenta síndrome de abstinência. & Oral & 3 \\
& Parenteral + nasal & 1 \\
\hline Caso 9. T. P.., 25 anos de idade, masculino. Foi & Oral & 1 \\
tomar água na mangueira do jardim e engoliu um & Mordedura/picada & 20 \\
filhote de cobra. Achou outros filhotes dentro da & Oral + mordedura/picada & 2 \\
mangueira. Identificados pelo pessoal do Centro de & Outra *** & 1 \\
Intoxicações como filhote de Cruzeira (Bothrops). & & 1 \\
\hline
\end{tabular}

* Couro cabeludo; ${ }^{* *} 9$ Transplacentária, 7 placenta, hemoplacentária, precisa de mais especificação, sem descrição.

*** $\mathrm{N}$ ão especificada. 
Ressalta-se ainda que a rotatividade de profissionais na atividade de registro de casos é grande, pois os plantonistas trabalham em escalas e 0 tempo de interação das equipes, entre um plantão e outro, émínimo, o que dificulta o processo comunicativo.

Os resultados encontrados demonstram falta de um entendimento uniforme por parte dos centros nos casos abordados, uma vez que várias categorias são assinaladas para um mesmo caso. Isso ocorre mesmo quando as variáveis e respectivas categorias referentes aos casos tratados no presente estudo apresentam suas definições contempladas nos materiais de consulta dos centros, como as Monografias de Toxicologia de Urgência (MTU) e o M anual de Preenchimento da Ficha de N otificação e de Atendimento.

Mesmo sendo um material comum de consulta dos centros no atendimento dos casos, amplamente distribuído e divulgado na rede de centros ${ }^{9}$, as MTU não minimizam o problema de divergência no processo de notificação, como pode ser observado na seção de resultados ao descrever os casos 1, 2 e 3 (Quadro 1).

O M anual dePreenchimento da Ficha deAtendimento e Notificação foi elaborado por representantes dos centros em uma reunião na Fundação O swaldo Cruz, no ano de2001, sendo acordado pelos membros da rede de centros. As informações contidas neste documento deveriam, teoricamente, ser colocadas em prática. Seriaaconselhável também que a equipe de plantonistas responsável pelo atendimento e pela notificação dos casos de intoxicação eenvenenamento, além deterem esse manual sempre por perto, recebessem treinamento para que o mesmo fosse usado adequadamente. Contudo, foi verificado, pelos resultados obtidos nesteestudo, um baixo índice de uso deste manual, como pode ser observado pela descrição dos casos 4, 5 e 6 (Quadro 2). Tais resultados estão em consonância com os seguintes depoimentos: Esse centro não utiliza nenhum manual para orientar o preenchimento das fichas denotificação. N em sabia da existência do manual quefoi acordado em uma reunião na Fiocruz. (Entrevistado X)

$O$ centro não possui manual, só a ficha. Não conheço o manual que foi acordado, só a ficha. $\mathrm{N}$ ão existe treinamento para o profissional que preenche a ficha. 0 coordenador fala sempre conosco no sentido de melhorar os dados estatísticos, mas não existe um curso para isso, uma capacitação. (Entrevistado Y)

Tais fatos geram preocupação dos próprios centros com a confiabilidade dos dados gerados, o que pode ser verificado no depoimento a seguir: Para a codificação da informação, ela (a ficha) quase que atende, a gente tem que avaliar mais um pouco essas classes, padronizar, porque é complicado. Ela não atende porque você não sabe onde vai colocar (não sabe classificar o caso dentre as categorias existentes). (Entrevistado $Y$ )

Chama a atenção 0 alto grau de dispersão no uso das categorias na notificação dos casos apresentados aos centros. Essa visão múltipla dos eventos revela a necessidade de padronização dos procedimentos adotados e do processo de notificação dos casos.

A tarefa de se obter registrosindividuais confiáveis écomplexa, como aponta Senra ${ }^{10}$, pois no processo de coleta de dados, há um número grande e heterogêneo de pessoas envolvidas; portanto, a qualidade das informações geradas depende da qualidade dos profissionais envolvidos nesta etapa. Além de um bom treinamento da equipe, deve haver uma conscientização por parte dos profissionais sobre a importância do trabalho no qual estão envolvidos.

Os dados somente serão gerados com precisão se forem definidos padrões, que de acordo com a definição da Organização Internacional de Padronização (International Standards O rganization - ISO), citado por Leão ${ }^{11}$, é um documento estabelecido por consenso e aprovado por um grupo reconhecido, que consolida para uso geral erepetido um conjunto de regras, protocolos ou características de processos com o objetivo de ordenar e organizar atividades em contextos específicos para o benefício de todos.

González de Gómez ${ }^{12}$ associa o padrão à função de gerar acordos e compromissos entre as práticas de definição ede classificação de diferentes grupos, eque implicam a aceitação de valores e de regras reguladoras dos acordos pelos diferentes grupos que deles fazem uso.

Em relação aos eventos polêmicos, que não estão previstos, acredita-se que o processo comunicativo entre os diversos centros da rede resolveria, pois a decisão tomada seria consensual eentão poderia se definir um padrão, legitimado por todos.

A discussão entre os representantes dos centros, no sentido de acordar determinada categorização em relação às variáveis que são registradas na ficha, possibilitará a geração de padrões, 0 que é imprescindível para que as informações possam ser geradas com o maior nível de qualidade possível. Porém, nada acontecerá se os padrões gerados não forem legitimados pelo grupo.

Nesse sentido, o processo comunicativo ga- 
nha uma importância fundamental, pois em grupos heterogêneos e com grande número de pessoas, o processo para se chegar a um consenso é muito complexo e, muitas vezes, resolvido por maioria enão por unanimidade, o quetorna mais importanteque todos quefazem partedessegrupo assumam o compromisso de aplicar o acordo estabelecido, tendo neste momento uma visão sistêmica e entendendo que a interferência pessoal em uma fase desse processo influenciará no resultado final.

O processo de implantação dos padrões é, sem dúvida, muito trabalhoso. A decisão da rede deverá ser colocada em prática e, nesse aspecto, a área gerencial de recursos humanos de cada centro poderá auxiliar na divulgação e treinamento dos procedimentos a serem adotados, contribuindo para a uniformidade nos registros dos casos deintoxicação eenvenenamento emelhorando de forma significativa seu aspecto qualitativo.

Como severificou, no momento da coleta de dados, devido ao grande número de pessoas envolvidas, a necessidade de uma padronização nos procedimentos desta etapa do trabalho é fundamental. N esta fase, a grande preocupação é com o conteúdo e não com a forma. É preciso tornar visível o que está invisível, como afirmam Lara et al. ${ }^{13}$, em sua discussão de metassistema de informações estatísticas, "[...] énecessário compatibilizar pontos de vista nem sempre homogêneos".

Independente do uso de tecnologias para captura e processamento, o registro de dados no setor de coleta responde por um processo de suma importância. É do nascedouro, dos inputs, que depende parte significativa da robustez de qual quer sistema de informação. M ota et al. ${ }^{2}$ ressaltam a relevância do conjunto deinstrumentos (fichas de notificação e outros) usados para registro earmazenamento de dados - deles dependem a precisão na recuperação e o cumprimento, em tempo hábil, do fluxo de dados atéo processamento, a consolidação, análise e difusão. Portanto, o grau de confiança da informação gerada nesse processo depende desses primeiros registros, que Senra ${ }^{10}$ chama de inscrições e descrições de primeira ordem.

A combinação de recursos tecnológicos ehumanos de qualidade é um fator determinante para a obten ção deinformações confiáveis, como ressalta Senra ${ }^{10}$, que mesmo com novos métodos e usando novas tecnologias em todo o processo de geração das estatísticas, os principais instrumentos ainda são as massas de seres humanos: para registrar, interpretar, classificar, contar, perguntar.
0 momento do registro de dados é muito importante, o que se não for feito com muita atenção e zelo, compromete a qualidade dos dados e consequentemente a confiabilidade das informações que são geradas a partir deles.

Apesar de todo o apoio da tecnologia e do desenvolvimento de metodologias no sentido de se gerar informação com qualidade e no tempo adequado, isto pouco adianta se os dados não forem gerados com o mesmo espírito, com atenção e cuidado, pois é a partir deles que uma informação pode se tornar confiável, ou não.

\section{Considerações finais}

Este estudo visou contribuir para a melhoria da qualidade das informações produzidas pelo SINITOX, sob a perspectiva da padronização do instrumento de coleta de dados, o qual evidenciou, de forma clara, uma fragilidade nesse instrumento e abriu a possibilidade de se propor a criação de padrões pactuados por todos para que as fichas denotificação sejam preenchidas deforma uniforme por profissionais devidamentetreinados e conscientizados quanto à importância do trabalho, utilizando manuais com orientações claras para esse preenchimento.

Pode-se considerar como uma das razões para o problema da padronização do registro desses casos a própria criação dos centros, que foi um processo isolado epontual, cada um com vínculos institucionais diferentes, maneiras diferentes de trabalho, perfis diferentes. Porém, atualmente, fazem parte de uma grande rede nacional e, para tanto, precisam compor uma unidade, pela necessidade de comunicação que é central no estabelecimento de redes.

Os resultados abordados deixam bem claro que a notificação desses casos não segue um padrão, tanto em relação às diversas fichas de notificação utilizadas eo não uso do manual para orientar o preenchimento, quanto ao próprio entendimento pelos profissionais que atuam nesta fase de registro dos dados, podendo acarretar um comprometimento qualitativo nos dados e na informação gerada posteriormente no SIN ITOX.

A exemplo do Sistema de Informações sobre Mortalidade (SIM), que ao adotar um modelo único padronizado da declaração de óbitos permitiu a uniformização dos dados e a apuração das informações de interesse para o setor saúde ${ }^{14}$, acredita-se que 0 aprimoramento e a padronização do instrumento de coleta de dados atualmente utilizado pelos CIAT possam propiciar um en- 
tendimento uniforme pelos profissionais envolvidos no processo de notificação, além de também favorecer a geração de dados mais fidedignos para uma melhor análise dos casos de intoxicação e envenenamento ocorridos no país.

É importante ressaltar que, antes mesmo de se avançar neste sistema, com foco em soluções tecnológicas, questões importantes precisam ser resolvidas. Os dados que alimentam o sistema devem possuir um grau de qualidade suficiente para que possam gerar indicadores que possibilitem o conhecimento da realidade. Paratanto, deve se dar a importância devida ao preenchimento correto dos dados e ao processo de padronização, que por sua vez pode minimizar as diferenças encontradas na geração das informações.

\section{Colaboradores}

RAL Santana participou de todas as etapas de elaboração do artigo; R Bochner colaborou na análise dos dados, discussão e revisão do artigo e M CS Guimarães colaborou na revisão da literatura, definição da metodologia do estudo e revisão do artigo.

\section{Referências}

1. Caldas LQA, Moraes ACL, Unes A. Toxicologia $M$ édica: a desinformação é alarmante. Ciência $\mathrm{H}$ oje 1996; 21:66-70

2. Mota $E$, Carvalho DM. Sistemas de Informação em Saúde. In: Rouquayrol MZ, Almeida Filho N, organizadores. Epidemiologia \& Saúde. 5a ed. Rio de Janeiro: MEDSI; 1999. p. 505-521.

3. Angeloni $M T$. Elementos intervenientes na tomada de decisão. Ci. Inf. 2003; 32(1):17-22.

4. Agência Nacional de Vigilância Sanitária. Rede Nacional de Centros de Informação e Assistência Toxicológica: proposta de Regulamentação dos Centros de Informação e Assistência Toxicológica. Brasília: GGTOX; 2004. [M imeo]

5. Yin RK. Estudo de caso - planejamento e métodos. $2^{\mathrm{a}}$ ed. Porto Alegre: Bookman; 2001.

6. Stake RE. The case study method in social inquiry. Educational Researcher 1978; 7(2):5-8.

7. Erskine JA, Leenders M R, M auffette-Leenders LA. Teaching with cases. Ontario: University of Western Ontario; 1981.

8. Centro de Informação Toxicológica do Rio Grande do Sul. M onografias de Toxicologia de U rgência. Porto Alegre: ATOX; 1997.

9. Fundação Oswaldo Cruz. Centro de Informação Científica e Tecnológica. Sistema Nacional de Informações Tóxico-Farmacológicas. M anual de Preenchimento da Ficha de $\mathrm{N}$ otificação e de Atendimento: Centros de Assistência Toxicológica. Rio de Janeiro: Fiocruz; 2001. 
10. Senra NC. A Coordenação da Estatística Nacional: 0 equilíbrio entre o desejável e o possível [tese]. Rio de Janeiro (RJ): Escola de Comunicação, Universidade Federal do Rio de Janeiro; 1998.

11. Leão BF. Padrões para representar a informação em saúde. In: Seminário Nacional de Informação e Saúde, 1 . 0 setor saúde no contexto da sociedade da informação. Rio de Janeiro: Fiocruz; 2000. p. 21-34.

12. González de Gómez MN. Da política de informação ao papel da informação na política. Rev Internacional de Estudos Políticos 1999; 1(1):67-93.

13. Lara M LG, Camargo JCC, Rocha SG. Informação estatística e cidadania. São Paulo em Perspectiva 2002; 16(3):86-91.

14. M ello Jorge MHP, Laurenti R, Gotlieb SLD. Análise da qualidade das estatísticas vitais brasileiras: a experiência de implantação do SIM e do SINASC. Cien Saude Colet 2007; 12(3):643-654.

Artigo apresentado em 15/08/2008

Aprovado em 29/01/2009

Versão final apresentada em 21/02/2009 